\title{
Reanimación cardiopulmonar básica: conocimiento teórico, desempeño práctico y efectividad de las maniobras en médicos generales
}

\author{
LUIS ROJAS ${ }^{1}$, ANDRÉS AIZMAN $^{1}$, JUAN PABLO ARAB $^{3}$, \\ FRANCO UTILI ${ }^{1}$, MAX ANDRESEN MsC ${ }^{2}$
}

\section{Basic cardiopulmonary resuscitation: knowledge, practical skills and effectiveness of maneuvers of general physicians}

Background: General physicians should be adequately trained to deliver effective resuscitation during ventricular fibrillation (VF). Aim: To assess the degree of knowledge, skills and practical effectiveness in cardiopulmonary resuscitation (CPR) of Chilean general physicians. Materials and Methods: Forty eight general physicians starting Anesthesiology or Internal Medicine residency programs were evaluated. They answered a modified American Heart Association Basic Life Support Course written test and individually participated in a witnessed VF cardiac arrest simulated scenario. Execution of resuscitation tasks in the correct order, the quality of the maneuvers and the use of defibrillator were registered. Results: All participants acknowledged the importance of uninterrupted CPR and early defibrillation. Seventy five percent knew the correct frequency of chest compressions, but only $6.25 \%$ knew all the effective chest compression characteristics. Ninety eight percent knew the recommended number of breaths per cycle. In practice, 58\% performed effective ventilations, 33\% performed uninterrupted compressions, $14 \%$ did them with adequate frequency and only $8 \%$ performed chest compressions adequately. Forty four percent requested a defibrillator within 30 seconds and $31 \%$ delivered the first defibrillation within 30 seconds of defibrillator arrival. Airway, breathing, circulation and defibrillation sequence was correctly performed by $12 \%$ of participants and $80 \%$ acknowledged that their medical training was inadequate or insufficient for managing a cardiac arrest. Conclusions: Despite an elevated degree of knowledge about key aspects of CPR, this group of Chilean physicians displayed suboptimal practical skills while performing $C P R$ in a simulated scenario, specially delivering effective chest compressions and promptly asking for and using the defibrillator.

(Rev Med Chile 2012; 140: 73-77).

Key words: Cardiopulmonary resuscitation; Electric countershock; Ventricular fibrillation.

'Escuela de Medicina.
Departamento de Medicina
Interna. Facultad de
Medicina. Pontificia
Universidad Católica de
Chile.
Escuela de Medicina.
Departamento de Intensivo.
Facultad de Medicina.
Pontificia Universidad
Católica de Chile.
3Interno. Escuela de
Medicina. Pontificia
Universidad Católica de
Chile.
Recibido el 30 de mayo de
2011, aceptado 27 octubre
2011.
Correspondencia a:
Dr. Luis Rojas
Departamento de
Medicina Interna. Facultad
de Medicina Pontificia
Universidad Católica de
Chile.
Marcoleta \#367, 50 piso.
Fono: 3543151
Fax: 6329213
E-mail: Irojas@med.puc.cl
Dr. Max Andresen.
Departamento de Intensivo.
Facultad de Medicina
Pontificia Universidad
Católica de Chile.
Marcoleta \# 367,4 Piso
UPC.
Fono: 3543265
E-mail: andresen@med.
puc.cl

L a enfermedad coronaria es la primera causa de muerte en el mundo ${ }^{1,2}$. La manifestación más severa es la muerte súbita (MS), representando más de $60 \%$ de estas muertes ${ }^{3-5}$. Se estima que la fibrilación ventricular (FV) es el ritmo electrocardiográfico más frecuente durante la MS, siendo su principal tratamiento la reanimación cardiopulmonar (RCP) efectiva y la desfibrilación precoz. La RCP básica aumenta hasta en 4 veces el éxito de la desfibrilación ${ }^{6}$. Así, es de vital 
importancia que los equipos médicos tengan los conocimientos y destrezas en RCP para mejorar las expectativas de sobrevida y recuperación funcional de estos pacientes.

Son pocos los estudios que han intentado evaluar los conocimientos y destrezas del personal médico en RCP y la mayor parte de ellos ha demostrado malos resultados ${ }^{7,8}$. Existe sólo un trabajo al respecto en nuestro medio publicado hace casi 20 años. Dicho estudio evaluó sólo conocimientos teóricos de 41 médicos y 30 internos de séptimo año usando una prueba de elección múltiple. Ésta sólo fue aprobada por 39\% de los médicos y 10\% de los internos 9 . Parece necesario instaurar métodos más eficaces de instrucción, donde el empleo de escenarios clínicos simulados puede ser una herramienta útil para mejorar tanto el aprendizaje como la evaluación de las competencias terminales del egresado de medicina.

El objetivo del presente estudio fue evaluar el nivel de conocimientos teóricos y destrezas prácticas en RCP en una muestra de médicos generales de nuestro país.

\section{Material y Método}

Se realizó un estudio de corte transversal donde se evaluaron la totalidad de médicos generales que estaban ad portas de iniciar un programa de especialización en Anestesiología y Medicina Interna en el Hospital Clínico de la Pontificia Universidad Católica de Chile. El número de médicos fue 48 y eran egresados de distintas universidades chilenas.

Para evaluar competencias cognitivas se realizó un test escrito (individual y anónimo) de múltiple elección, confeccionado en base a las guías clínicas de RCP de la American Heart Association (AHA) del año $2005^{10}$. Las preguntas del test fueron validadas en dos aspectos: 1) El contenido: mediante cotejo entre referentes técnicos; 2) La forma: el cuestionario fue aplicado a 30 personas semejantes a la muestra que fue encuestada para así evaluar la comprensión de la redacción de las preguntas y el vocabulario usado. Los tests escritos fueron corregidos por evaluadores externos.

Para evaluar competencias prácticas, los médicos participaron en un escenario simulado de PCR por fibrilación ventricular en una sala doble espejo. Se empleó un maniquí de simulación modelo Laerdal SimMan ${ }^{\circledast}$ acoplado a un computador que permite emular el ritmo cardiaco y registrar las maniobras de reanimación realizadas. También se registraron las maniobras de RCP mediante cámaras de vídeo. Los médicos recibieron la instrucción de asistir a un paciente inconsciente en un box de urgencia de un centro asistencial, para lo cual contarían con el apoyo de un auxiliar paramédico y de instrumental básico (fuente de oxígeno, fonendoscopio, esfigmomanómetro, dispositivo de bolsa-válvula-mascarilla). Se dispuso fuera del box de un carro de paro equipado con un desfibrilador monofásico, el cual fue entregado por el auxiliar paramédico sólo en caso de que el médico lo solicitara.

Un equipo de 4 expertos, compuesto por médicos intensivistas y hospitalistas confeccionó una pauta de evaluación validada mediante técnica Delphi ${ }^{11}$. El panel de expertos identificó los aspectos de importancia en la pauta de evaluación, guiados por las recomendaciones de International Liaison Committee on Resuscitation (ILCOR) ${ }^{12}$. Así, mediante los registros se evaluó: evaluación del nivel de conciencia, solicitud de ayuda a terceros, evaluación de la presencia signos de circulación (respiración espontánea y pulso arterial carotideo), postura del operador y sus brazos, amplitud y frecuencia del masaje cardiaco, ejecución ininterrumpida de éste, frecuencia y efectividad de la ventilación asistida, solicitud precoz y uso adecuado del desfibrilador, el tiempo trascurrido antes de la primera descarga y seguimiento de la secuencia de reanimación $\mathrm{ABCD}$.

En la evaluación de los signos de circulación se observó la apertura correcta de la vía aérea, la búsqueda de respiración espontanea (escuchar, sentir y ver la respiración) y del pulso arterial en las arterias carotideas. La solicitud de ayuda se consideró correcta cuando esta consideraba al menos la solicitud de un carro de paro o desfibrilador. Las compresiones torácicas fueron consideradas efectivas cuando: eran realizadas a una frecuencia de 100 a 120 compresiones por minuto ininterrumpidas y con una profundidad de 3,5 a $5 \mathrm{~cm}$. Las ventilaciones a su vez, se consideraron efectivas cuando se realizaban con un intervalo de 2 por cada 30 compresiones torácicas, si lograban elevar el tórax del maniquí y daban tiempo para la salida de aire (aproximadamente 2 segundos). La solicitud del desfibrilador debía ocurrir antes de 30 segundos de haber evaluado al paciente y descarga eléctrica antes de 30 segundos de haber 
Reanimación cardiopulmonar básica. Evaluación en médicos generales - L. Rojas et al

evaluado el ritmo (FV) en el monitor. Se consideró correcto cuando la energía seleccionada antes de la descarga era de 360 jouls.

Los vídeos y el registro computacional del maniquí fueron analizados por evaluadores externos mediante pautas de cotejo, las cuales fueron validadas de la misma forma que lo fueron las pruebas escritas.

El estudio contó con la aprobación del comité de ética local.

\section{Resultados}

Los resultados del test escrito teórico mostraron que $100 \%$ de los médicos generales reconoció la importancia de la RCP ininterrumpida y desfibrilación precoz. De ellos $75 \%$ conoce la frecuencia recomendada de compresiones torácicas y sólo $6,25 \%$ conoce todas las características que requieren las compresiones torácicas efectivas. El 98\% sabe que la principal acción para recuperar la circulación es la desfibrilación.

En la situación clínica simulada, prácticamente todos evaluaron primero el nivel de conciencia, solicitaron ayuda, sin embargo, el resto de la secuencia de maniobras fue seguida adecuadamente por $12 \%$ de de los evaluados. El 33\% realizó compresiones torácicas ininterrumpidas, pero sólo $8 \%$ ejecutó compresiones torácicas efectivas. Sólo $58 \%$ realizó ventilaciones adecuadas. Respecto al uso del desfibrilador, $41 \%$ de los médicos lo solicitó antes de los 30 segundos, la mitad seleccionó adecuadamente la energía de descarga y sólo 31\% lo utilizó precozmente (Tabla 1).

El 80\% de los médicos generales evaluados en este estudio reconoció no sentirse suficientemente preparados para atender un PCR con la formación médica recibida en pregrado.

\section{Discusión}

La resucitación cardiopulmonar efectiva y la desfibrilación precoz han sido ampliamente demostradas como terapia efectiva frente a la muerte súbita por fibrilación ventricular ${ }^{6,13}$.

En Estados Unidos la Accreditation Council for Graduate Medical Education - ACGME definió la $\mathrm{RCP}$ como una de las competencias genéricas de los egresados de Medicina ${ }^{14}$. En nuestro país, la enseñanza de RCP es parte de la malla curricular de las principales Escuelas de Medicina. Sin embargo, la certificación de la adquisición de dichas destrezas, no son un requisito para trabajar en muchos centros asistenciales.

El único estudio a nivel nacional que evaluó los conocimientos de resucitación cardiopulmonar en médicos e internos de Uribe $M$. et al publicado en $1992^{9}$, mostró una severa falta de conocimientos entre los evaluados. Casi 20 años después la situación no es muy distinta. Si bien el nivel de conocimientos teóricos parece haber mejorado, existen severas deficiencias en la aplicación práctica de éstos, especialmente en aquellas maniobras más importantes para una RCP exitosa (compresiones torácicas efectivas y solicitud y uso del desfibrilador). Probablemente esto obedezca a la falta de exposición de los estudiantes a situaciones clínicas (reales o simuladas) en donde puedan aplicar dichas destrezas.

Diversos artículos han abordado la problemática a nivel mundial. Se han realizado estudios en Inglaterra ${ }^{15}$, Estados Unidos de Norteamérica ${ }^{16}$,

Tabla 1. Resultados de la evaluación de las competencias prácticas de los médicos participantes

\begin{tabular}{|lc|}
\hline Destrezas evaluadas & $\begin{array}{c}\text { \% de } \\
\text { médicos }\end{array}$ \\
\hline $\begin{array}{l}\text { Verifica inconsciencia } \\
\text { Solicita ayuda }\end{array}$ & 98 \\
\hline Abre adecuadamente la vía área & 99 \\
Verifica presencia de respiración & 56 \\
\hline $\begin{array}{l}\text { Verifica presencia de pulso carotideo } \\
\text { Realiza } 2 \text { ciclos de } 30 \text { compresiones: }\end{array}$ & 76 \\
\hline ventilaciones en un minuto & 19 \\
\hline $\begin{array}{l}\text { Realiza compresiones torácicas con } \\
\text { frecuencia } 100 \text { a } 120 \text { x' } \\
\text { Realiza ventilaciones efectivas }\end{array}$ & 31 \\
\hline $\begin{array}{l}\text { Solicita el desfibrilador antes de los } \\
30 \text { segundos }\end{array}$ & 58 \\
\hline $\begin{array}{l}\text { Selecciona la energía de descarga } \\
\text { correcta }\end{array}$ & 41 \\
\hline $\begin{array}{l}\text { Realiza descarga eléctrica antes de } \\
\text { Sigue la secuencia ABCD adecuadamente }\end{array}$ & 56 \\
\hline
\end{tabular}


Japón ${ }^{17}$, Nueva Zelanda ${ }^{18}$, Suecia ${ }^{19}$ y China $^{20}$ llegando a la misma conclusión: existe falta de entrenamiento en RCP en los programas de educación médica de pregrado, determinando que los médicos recién egresados no se sientan cómodos con la reanimación y cometan errores graves ${ }^{16,21-23}$. Del mismo modo, distintos reportes muestran que los estudiantes de medicina consideran importante y necesario mayor entrenamiento en habilidades prácticas de reanimación ${ }^{24,25}$. Además, se conoce que el decaimiento del conocimiento teórico comienza ya a partir de las seis semanas de finalizado un curso y que progresa con el tiempo ${ }^{26,27}$.

Una de las limitaciones de nuestro estudio es que evaluó a una población seleccionada de médicos no especialistas no necesariamente representativa del universo total, sin embargo, tiene la fortaleza de contar con médicos egresados de distintas universidades del país, tanto públicas como privadas. El grupo de médicos evaluados superó un proceso de selección riguroso en base a su buen desempeño académico en pregrado. El posible sesgo que esto haya introducido en nuestros resultados hace suponer que el desempeño general puede ser aun peor.

En suma, los médicos generales evaluados reconocen la importancia de una RCP básica efectiva y una desfibrilación precoz. Sin embargo, existen deficiencias marcadas en el desempeño práctico. La mayoría de los médicos evaluados reconoce a su vez que su formación médica de pregrado fue insuficiente en RCP.

Debido a lo anterior y a que el éxito de la reanimación depende de maniobras bien realizadas, es necesario reforzar las destrezas en RCP y su mantención en el tiempo durante la formación médica ${ }^{28}$. Como fue demostrado en nuestra experiencia publicada recientemente respecto a intubación endotraqueal ${ }^{29}$, la metodología utilizada hoy en día no asegura la adquisición de este tipo de competencias en esta etapa curricular, siendo necesario instaurar métodos más eficaces de instrucción, donde el empleo de escenarios clínicos simulados puede ser una herramienta útil para mejorar el aprendizaje y evaluación de las competencias terminales del egresado de medicina.

Agradecimientos. Queremos agradecer el apoyo brindado por los Médicos Pablo Hasbún, Gerty Barrios y Marcos Rattalino en la ejecución de este estudio y al personal del Centro Médico
San Joaquín de la Red de Salud de la Pontificia Universidad Católica de Chile que hicieron posible la implementación de cada una de las etapas del proyecto.

\section{Referencias}

1. Levi F, Lucchini F, Negri E, La Vecchia C. Trends in mortality from cardiovascular and cerebrovascular diseases in Europe and other areas of the world. Heart 2002; 88: 119-24.

2. Murray CJ, López AD. Mortality by cause for eight regions of the world: Global Burden of Disease Study. Lancet 1997; 349: 1269-76.

3. Handley AJ, Koster R, Monsieurs K, Perkins GD, Davies $\mathrm{S}$, Bossaert L. European Resuscitation Council guidelines for resuscitation 2005. Section 2. Adult basic life support and use of automated external defibrillators. Resuscitation 2005; 67S1: S7-23.

4. Nolan J. European Resuscitation Council guidelines for resuscitation 2005. Section 1. Introduction. Resuscitation 2005; 67S1 1: S3-6.

5. Zheng ZJ, Croft JB, Giles WH, Mensah GA. Sudden cardiac death in the United States, 1989 to 1998. Circulation 2001; 104: 2158-63.

6. Tan EC, Severien I, Metz JC, Berden HJ, Biert J. First aid and basic life support of junior doctors: A prospective study in Nijmegen, the Netherlands. Med Teach 2006; 28: 189-92.

7. Holmberg M, Holmberg S, Herlitz J. Effect of bystander cardiopulmonary resuscitation in out-of-hospital cardiac arrest patients in Sweden. Resuscitation 2000; 47: 59-70.

8. Jansen JJ, Berden HJ, van der Vleuten CP, Grol RP, Rethans J, Verhoeff CP. Evaluation of cardiopulmonary resuscitation skills of general practitioners using different scoring methods. Resuscitation 1997; 34: 35-41.

9. Uribe M, Bianchi V, Carvajal C, Kauffman R. [Assessment of knowledge of cardiopulmonary resuscitation]. Rev Med Chile 1992; 120: 1231-4.

10. American Heart Association Guidelines for Cardiopulmonary Resuscitation and Emergency Cardiovascular Care. Circulation 2005; 112SIV: IV-1-IV 211.

11. Hasson f, Keeney S, Mckenna H. Research guidelines for the Delphi survey technique. J Adv Nurs 2000; 32(4): 1008-15.

12. Chamberlain DA, Hazinski MF; European Resuscitation Council; American Heart Association; Heart and Stroke Foundation of Canada; Resuscitation Council of Southern Africa; Australia and New Zealand Resuscita- 
tion Council; Consejo Latino-Americano de Resusucitación. Education in resuscitation: an ILCOR symposium: Utstein Abbey: Stavanger, Norway: June 22-24, 2001. Circulation 2003; 108: 2575-94.

13. Accreditation council for graduate medical education. [homepage en Internet]. Chicago: ACGME;c2000-2009. Outcome Project; Disponible en: http://www.acgme.org/ acWebsite/home/home.asp. [consultado el 8 de noviembre de 2010].

14. Ramaraj R, Ewy GA. Rationale for continuous chest compression cardiopulmonary resuscitation. Heart 2009; 95: 1978-82.

15. Seedat A, Walmsley H, Rochester S. Advanced life support (ALS) and medical students: do we feel confident and competent after completing our training? Resuscitation 2008; 78: 100-1.

16. Smith CM, Perkins GD, Bullock I, Bion JF. Undergraduate training in the care of the acutely ill patient: a literature review. Intensive Care Med 2007; 33: 901-7.

17. Suzuki A, Suzuki Y, Takahata O, Fujimoto K, Nagashima K, Mamiya K, et al. A survey of 3,303 6th-year medical students from 36 universities concerning knowledge of resuscitation--more than $80 \%$ of medical students can not perform standard cardiopulmonary resuscitation? Masui 2001; 50: 316-322.

18. Price CS, Bell SF, Janes SE, Ardagh M. Cardio-pulmonary resuscitation training, knowledge and attitudes of newly-qualified doctors in New Zealand in 2003. Resuscitation 2006; 68: 295-9.

19. Israelsson J, Källén P, Carlsson J. Test cases: in-hospital, scenario-based cardiopulmonary resuscitation training. Med Educ 2009; 43: 1099-100.

20. Caves ND, Irwin MG. Attitudes to basic life support among medical students following the 2003 SARS outbreak in Hong Kong. Resuscitation 2006; 68: 93-100.

21. Scott G, Mulgrew E, Smith T. Cardiopulmonary resuscitation: attitudes and perceptions of junior doctors. Hosp Med 2003; 64: 425-8.

22. Ahmed HU, Kellett C, Ashworth M, Nazir S. First aid and cardiopulmonary resuscitation training for medical students. Med Educ 2004; 38: 913.

23. Gillard JH, Dent TH, Jolly BC, Wallis DA, Hicks BH. CPR and the RCP (2). Training of students and doctors in UK medical schools. J R Coll Physicians Lond 1993; 27: 412-7.

24. Graham CA, Guest KA, Scollon D. Cardiopulmonary resuscitation. Paper 2: A survey of basic life support training for medical students. J Accid Emerg Med 1994; 11: 165-167.

25. Stross JK. Maintaining competency in advanced cardiac life support skills. JAMA 1983; 249: 3339-41.

26. Young R, King L. An evaluation of knowledge and skill retention following an in-house advanced life support course. Nurs Crit Care 2000; 5: 7-14.

27. Su E, Schmidt TA, Mann NC, Zechnich AD. A randomized controlled trial to assess decay in acquired knowledge among paramedics completing a pediatric resuscitation course. Acad Emerg Med 2000; 7: 779-86.

28. Chamberlain DA, Hazinski MF. Education in resuscitation: an ILCOR symposium: Utstein Abbey: Stavanger, Norway: June 22-24, 2001. Circulation 2003; 108: 257594.

29. Andresen M, Riquelme, Hasbún P, Díaz C, Montaña R, Regueira T. Evaluación de competencias de intubación traqueal mediante un escenario simulado en internos de medicina. Rev Med Chile 2011; 139: 165-70. 